\title{
FATORES ETIOLÓGICOS DO BRUXISMO DO SONO: REVISÃO DE LITERATURA
}

\section{ETIOLOGY OF SLEEP BRUXISM: LITERATURE REVIEW}

\author{
Mariana Fernandes Calderan* \\ Thiago Cruvinel Silva* \\ Daniela Rios Honório*** \\ Thais Marchini Oliveira*... \\ Maria Aparecida de Andrade Moreira Machado
}

\begin{abstract}
RESUMO
Inúmeros estudos trazem o bruxismo como tema central, relacionando-o com vários distúrbios, tais como problemas dentários, problemas do sono, dores de cabeça e nos músculos da face. Para compreendermos profundamente essa atividade muscular que o bruxismo provoca, temos que compreender seus fatores etiológicos. Porém, o bruxismo é multifatorial e alguns estudos têm demonstrado quais fatores estão comumente associados a ele. O objetivo deste trabalho é realizar uma revisão de literatura, analisando quais fatores etiológicos são mais comumente citados em estudos sobre o bruxismo do sono.
\end{abstract}

Descritores: Bruxismo do sono, Etiologia, Transtornos do sono

\section{ABSTRACT}

Studies having bruxism as main theme relate it to many disturbs, such as dental problems, sleep problems, headaches and facial muscle pain. The etiology is important to deeply understand the muscular activities originated by the bruxism. However, bruxism is multifactorial and some common factors are often associated. The purpose of this study is to make a literature review that analyzes which etiological factors are more often reported in sleep bruxism studies.

Descriptors: Sleep bruxism, Etiology, Sleep disorders.

* Mestranda em Ciências Odontológicas Aplicadas, área de concentração Odontopediatria, pela Faculdade de Odontologia de Bauru-USP. marifcalderan@ hotmail.com

** Professor Doutor do departamento de Odontopediatria, Ortodontia e Saúde Coletiva da Faculdade de Odontologia de Bauru-USP. Disciplina Odontopediatria. thiagocruvinel@fob.usp.br

*** Professora Associada do departamento de Odontopediatria, Ortodontia e Saúde Coletiva da Faculdade de Odontologia de Bauru-USP. Disciplina Odontopediatria. danirios@usp.br

**** Professora Doutora do departamento de Odontopediatria, Ortodontia e Saúde Coletiva da Faculdade de Odontologia de Bauru-USP. Disciplina Odontopediatria. marchini@usp.br

***** Diretora da Faculdade de Odontologia de Bauru e Professora Titular do departamento de Odontopediatria, Ortodontia e Saúde Coletiva da Faculdade de Odontologia de Bauru-USP. Disciplina Odontopediatria. mmachado@fob.usp.br 
CALDERAN MF

$S I L V A T C$

HONÓRIO DR

OLIVEIRA TM

MACHADO MAAM

FATORES

ETIOLÓGICOS

DO BRUXISMO DO

SONO: REVISÃO DE

LITERATURA

\section{4}

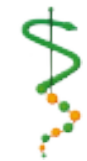

REV, ODONTOL.

UNIV. CID, SÃO

PAULO

$2014 ; 26(3): 243-$

9, SET-DEZ

\section{N T R O D U ÇÃ O}

O bruxismo é uma atividade repetitiva dos músculos mandibulares que envolve o ranger e apertar dos dentes e/ou o esfregar ou o impulsionar da mandíbula ${ }^{1}$. Ele pode ocorrer com crianças, jovens e adultos nas diferentes faixas etárias e manifesta-se de duas formas diferentes: o bruxismo de vigília, que ocorre durante o dia, e o bruxismo do sono, que acontece durante o período noturno ${ }^{2,3,4,5,6}$.

O ranger dos dentes é mais comum no bruxismo do sono, enquanto o apertar dos dentes é mais comum no bruxismo de vigília $^{7,8}$. Sua prevalência é de $7-15 \%$ em crianças e de $15-20 \%$ em adultos ${ }^{9}$, com maior ocorrência no gênero feminino ${ }^{7}$, ${ }^{10}$. O diagnóstico clínico do bruxismo do sono é realizado pelo autorrelato do paciente, ou relato de irmãos, parceiros ou pais que escutam o ranger dos dentes durante a noite juntamente com a presença de sinais e sintomas ${ }^{11}$. Os sinais e sintomas do bruxismo como hipertrofia dos músculos masseter e temporal, desgastes dentários, dor à palpação dos músculos mandibulares e relato de dor de cabeça ao acordar podem ser clinicamente analisa$\operatorname{dos}^{12,13}$. Outros exames para diagnóstico do bruxismo são utilizados e considerados mais confiáveis, tais como a Eletromiografia (EMG), que analisa os padrões e atividades musculares e a Polissonografia (PSG), que é apontada como padrão ouro para diagnóstico de bruxismo. Seu custo e aplicação dificultam seu uso ${ }^{11}$.

Estudos sobre os aspectos distintos do bruxismo, tais como sua definição, epidemiologia ${ }^{9,10}$, os efeitos causados sobre a saúde geral do paciente e suas atividades motoras têm crescido nos últimos anos. Entretanto, a etiologia do bruxismo ainda não está bem definida ${ }^{1,9,10,12,13}$. Diferentes fatores etiológicos e de risco são apontados, mas a maioria dos autores afirma que, embora ocorrências locais possam ter influência, a principal causa é psicossocial ${ }^{14}$.

O objetivo deste trabalho é revisar a literatura referente aos fatores etiológicos comumente associados ao bruxismo do sono.

$$
\text { METODOLOG I A }
$$

Critérios de inclusão e estratégia de busca
Foram selecionados estudos clínicos aleatorizados, estudos coorte prospectivos, caso-controle e revisões de literatura que apresentaram o objetivo de estudar e/ ou discutir os possíveis fatores etiológicos do bruxismo em adultos e crianças.

Os levantamentos bibliográficos foram realizados nas bases de dados Pubmed, Web of Science e Lilacs. Como estratégia de busca foi utilizada a seguinte combinação de unitermos: "bruxism" OR "sleep bruxism" OR "childhood sleep bruxism" OR "adult sleep bruxism" OR "bruxism etiology" OR "bruxism anxiety" OR "bruxism stress" OR "bruxism psychological factors". Foram consideradas referências publicadas entre os anos de 1974 e 2014, nos idiomas inglês e português.

\section{Etiologia do bruxismo do sono}

Durante muitos anos, acreditou-se que a origem do bruxismo estava ligada à existência de interferências dentárias capazes de alterar os padrões oclusais e musculares do paciente ${ }^{15,16,17,18,19}$. A indicação de tratamento era pautada na realização de ajustes oclusais, pela alteração dos padrões de contato dentário, visando à prevenção e ao controle do bruxismo ${ }^{18}$. Como não há evidências suficientes para comprovação da efetividade dos ajustes oclusais no gerenciamento do bruxismo, essa prática continua controversa ${ }^{20,21}$.

O conhecimento sobre a diversidade de fatores etiológicos promoveu uma abordagem terapêutica mais complexa. Entretanto, os fatores oclusais não podem ser completamente descartados da etiologia do bruxismo 22 .

\section{Despertar Breve do Sono}

Outro possível fator que poderia desencadear o bruxismo é a excitação motora durante o sono provocando o despertar repentino. Através do exame eletromiográfico (EMG) durante o sono, algumas características dos padrões de movimentos dos músculos faciais podem ser analisadas, dentre elas, a atividade muscular mastigatória rítmica (AMMR). A AMMR ocorre em aproximadamente $60 \%$ das pessoas sem bruxismo. Entretanto, essa atividade é três vezes mais intensa entre pacientes bruxômanos, com $70 \%$ mais de contrações musculares. Isso acontece devido à estimulação gerada durante o sono. 
Essa estimulação, além de gerar as atividades musculares, também está envolvida em casos de despertares breves durante o sono $^{11,12}$.

O despertar breve do sono tem duração em torno de 3 segundos, não retomando a consciência $^{23}$. Sua origem parece vir da ativação dos sistemas subcortical e reticular, que controlam as atividades motoras, autônomas e do tálamo ${ }^{24,25}$. Ao despertar, alterações dos sistemas cardíaco, autônomo e atividades musculares podem ser observadas. Hipoteticamente, a frequência do bruxismo está ligada à frequência das atividades rítmicas dos músculos mastigatórios, provocada por despertares ${ }^{26,27}$. Kato et al. ${ }^{28}$ T.Centre d'etude sur le Sommeil et des Rythmes Biologiques, Hopital du Sacre-Coeur de Montreal, Facultes des medecine et de medecine dentaire, Universite de Montreal, Quebec, Cana$\mathrm{da}^{4}$ (2003) avaliaram a resposta durante o sono de pacientes com e sem bruxismo, sob estimulação para o breve despertar. A estimulação foi realizada de duas diferentes maneiras: a) vibro-tátil e auditiva, em diferentes intensidades; b) auditiva, com duração de 1 segundo. Os resultados apontaram maiores níveis de AMMR com associação de ranger dos dentes em pacientes bruxômanos. Em contrapartida, os níveis de AMMR foram menores em indivíduos sem bruxismo, sendo que o ranger dos dentes não foi observado. Os resultados sugerem que o bruxismo pode ser uma resposta fisiológica aos despertares do sono. A Classificação Internacional das Desordens do Sono considera que o bruxismo do sono está associado com o despertar repentino ${ }^{5,28}$.

Sistema Nervoso Autônomo (SNA) e Neurotransmissores

Como demonstrado anteriormente, o bruxismo do sono faz parte de uma série de estimulações complexas do sistema nervoso autônomo (SNA), promovendo breves despertares e alterando os batimentos cardíacos, movimentos respiratórios e atividades musculares ${ }^{24-27}$.

A ocorrência de reações fisiológicas e/ou psicológicas pode alterar a homeostasia. O SNA promove a liberação de neurotransmissores com o intuito de restabelecer a homeostasia, o que também in- fluencia na supressão ou exacerbação dos movimentos do bruxismo ${ }^{29,} 30,31,32$. Após a evidência de que a administração de um precursor da dopamina (L-dopa) a um paciente bruxômano, com diagnóstico de Doença de Parkinson, diminuiu o ranger dos dentes, o sistema dopaminérgico começou a ser estudado como um possível desencadeador das reações musculares ${ }^{32}$. A dopamina poderia estar ligada à ativação da estimulação do SNA durante o sono $^{33}$. Níveis elevados de catecolaminas também foram detectados na urina de pacientes com bruxismo em relação aos pacientes sem bruxismo ${ }^{34}$.

\section{Fatores Psicossociais}

Fatores psicossociais, como o estresse e a ansiedade, também são indicados como fatores etiológicos do bruxismo ${ }^{35,36}$. Em resposta ao estresse, ocorre a ativação do SNA e do eixo hipotalâmico-hipofisário-adrenal (HHA), ocorrendo um aumento da frequência cardíaca e respiratória, da pressão arterial e dos níveis de glicocorticóides circulantes, tais como o cortisol ${ }^{30}$. Estudos demonstram que pacientes bruxômanos tendem a ser mais estressados e ansiosos que pacientes sem bruxismo ${ }^{31-36}$.

\section{Fatores Externos ou Secundários}

Outros fatores também podem implicar na etiologia do bruxismo. São considerados fatores de risco, tais como medicamentos, tabagismo, álcool, outras drogas e cafeína ${ }^{37,38,39}$. Sabe-se que alguns medicamentos que ativam o sistema cardiovascular podem desencadear estímulos e alterar a qualidade do sono ${ }^{40}$. Medicamentos para o tratamento de algumas doenças, como déficit de atenção e hiperatividade, transtornos de movimentos (Doenças de Parkinson e Huntington) e outras doenças $^{11,41,42,43}$.

Em fumantes, ocorre uma interação entre a nicotina e os mecanismos responsáveis pela realização dos movimentos realizados durante o bruxismo ${ }^{44}$. Consumo de álcool, drogas e cafeína causam alterações na atividade do Sistema Nervoso Central (SNC), podendo contribuir para o desenvolvimento do bruxismo $37-39,44$.

\section{Fatores Genéticos}

A associação entre os fatores genéticos e a presença de bruxismo começou a ser discutida após um estudo que continha
CALDERAN MF

SILVA TC

HONÓRIO DR

OLIVEIRA TM

MACHADO MAAM

FATORES

ETIOLÓGICOS DO

BRUXISMO DO

SONO: REVISÃO DE

LITERATURA

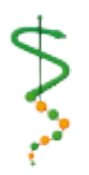

REV, ODONTOL.

UNIV. CID, SÃo

PAULO

2014; 26(3): $243-$

9 , SET-DEZ 
CALDERAN MF

SILVA TC

HONÓRIO DR

OLIVEIRA TM:

MACHADO MAAM

FATORES

ETIOLÓGICOS

DO BRUXISMO DO

SONO: REVISÃO DE

LITERATURA

246

REV, ODONTOL,

UNIV, CID, SÃO

PAULO

$2014 ; 26(3): 243-$

9, SET-DEZ uma amostra composta por irmãos gêmeos, monozigóticos e dizigóticos. Eles mostraram o mesmo padrão de mastigação e a mesma probabilidade de apresentarem o bruxismo ${ }^{12,45}$. A partir daí, outro estudo que também observou a relação do bruxismo em crianças gêmeas, com acompanhamento de 20 anos, tentou demonstrar a participação da genética como fator etiológico do bruxismo. Os resultados tendem a mostrar que nesses casos a genética pode estar envolvida com a presença de bruxismo ${ }^{46}$.

Apesar disso, há escassez de dados na literatura que comprovem a prevalência do bruxismo na família. É necessário que haja uma metodologia mais específica para se avaliar e diagnosticar o bruxismo. As metodologias aceitas para essa finalidade, são conseguidas através do diagnóstico realizado com o uso da polissonografia, da eletromiografia (EMG), do autorrelato de ranger os dentes e também da presença de sinais e sintomas clínicos ${ }^{11}$. Porém, muitos estudos levam apenas o diagnóstico clínico em consideração, causando, assim, à imprecisão do diagnóstico ${ }^{12}$. Também, ainda não foi descoberto nenhum tipo ou existência de um marcador genético, capaz de indicar a relação desses fatores com o desencadeamento do bruxismo ${ }^{11}$.

Portanto, assim como os fatores oclusais, os fatores genéticos precisam de meIhores evidências para contestar seu papel de agir sobre o bruxismo.

\section{Considerações Finais}

As evidências apontam que o bruxismo do sono é resultado da estimulação dos sistemas excitatórios e inibitórios (neurotransmissores) que tendem a alterar o equilíbrio entre o tronco cerebral e sistema de sono cortical ${ }^{11}$.

O bruxismo promove consequências no sistema estomatognático assim como pode estar ligado a um problema psicológico $^{14}$. Igualmente aos fatores etiológicos, o tratamento também não está definido. Devido à crescente epidemiologia dos casos de bruxismo, é importante conhecer as diferentes abordagens disponíveis para esses pacientes. Os tipos de tratamento que têm sido sugeridos envolvem as abordagens farmacológica, psicológica e odontológica. O tratamento farmacológico é geralmente indicado em casos severos e sempre em um curto período de tempo. Drogas do tipo benzodiazepínicos, anticonvulsivantes, beta-bloqueadores, agentes dopaminérgicos, antidepressivos e relaxantes musculares são as mais utilizadas ${ }^{47}$. Porém, ainda faltam evidências sobre a efetividade dessa conduta ${ }^{11}$. O tratamento psicológico baseia-se na terapia comportamental, na higiene do sono, no controle do estresse e em técnicas de relaxamento, mas não existem fortes evidências sobre nenhum desses tratamentos. $\mathrm{O}$ tratamento odontológico para o bruxismo inclui ajuste oclusal, restauração da superfície dentária, tratamento ortodôntico e placas oclusais ${ }^{47}$. Entretanto, dentre os tratamentos odontológicos disponíveis, as placas oclusais são as mais indicadas. Não foi comprovado que o uso das placas oclusais diminuam ou curem o bruxismo, seu uso é indicado priorizando a proteção das estruturas dentárias ${ }^{48}$. Cabe ao profissional e ao paciente decidirem quais condutas são melhor aplicadas em cada caso.

Não existem evidências suficientes na literatura sobre qual tratamento seria mais eficaz ou qual o fator etiológico do bruxismo. Sabe-se que ele está diretamente ligado à estimulação do SNA. Para conseguirmos realizar um tratamento efetivo do bruxismo, o conhecimento sobre sua etiologia deve estar consolidado. Para isso, é necessário que novos estudos clínicos randomizados sejam estabelecidos para descobertas de diretrizes que possam reduzir ou eliminar os sinais e sintomas-problemas advindos do bruxismo do sono. 
1. Lobbezoo F, Ahlberg J, Glaros AG, Kato T, Koyano K, Lavigne GJ, et al. Bruxism defined and graded: an international consensus. J Oral Rehabil 2013 Jan;40(1):2-4.

2. American Academy of Orofacial Pain. Guidelines for assessment, classification and management. Chicago: Quintessence; 1996.

3. Vanderas AP. Relationship between craniomandibular dysfunction and oral parafunctions in Caucasian children with and without unpleasant life events. J Oral Rehabil 1995 22(4):28994.

4. De Leeuw R. Orofacial pain: guidelines for assessment, diagnosis and management. 4 ed. Chicago: Quintessence; 2008.

5. American Academy of Sleep Medicine. International classification of sleep disorders, revised: diagnostic and coding manual. 2 ed. Chicago, Illinois: American Academy of Sleep Medicine; 2001.

6. The glossary of prosthodontic terms. J Prosthet Dent 2005 Jul;94(1):10-92.

7. Bayardo RE, Mejia JJ, Orozco S, Montoya K. Etiology of oral habits. ASDC J Dent Child1996 Sep-Oct;63(5):350-3.

8. Monaco A, Ciammella NM, Marci MC, Pirro R, Giannoni M. The anxiety in bruxer child. A case-control study. Minerva Stomatol 2002 Jun;51(6):24750.

9. Lavigne GJ, Manzini C, Kato T. Sleep bruxism: principles and practice of sleep medicine. 4 ed. Philadelphia: Elsevier Saunders; 2005.

10. Kato T, Lavigne GJ. Sleep bruxism: a sleep-related movement disorder. Sleep Med Clin 2010 5(1):9-35.

11. Carra MC, Huynh N, Lavigne G. Sleep bruxism: a comprehensive overview for the dental clinician interested in sleep medicine. Dent Clin North Am 2012 Apr;56(2):387-413.
12. Lavigne G, Manzini C, Huynh NT. Sleep bruxism. In: Kryger $\mathrm{MH}$, Roth $\mathrm{T}$, Dement WC. Principles and practice of sleep medicine. 5 ed. St Louis (MO): Elsevier Saunders; 2011. p. 1129-39.

13. Koyano K, Tsukiyama Y, Ichiki R, Kuwata T. Assessment of bruxism in the clinic. J Oral Rehabil 2008 Jul;35(7):495-508.

14. Bader G, Lavigne G. Sleep bruxism; an overview of an oromandibular sleep movement disorder. REVIEW ARTICLE. Sleep Med Rev 2000 Feb;4(1):2743.

15. Roark AL, Glaros AG, O'Mahony AM. Effects of interocclusal appliances on EMG activity during parafunctional tooth contact. J Oral Rehabil 2003 Jun;30(6):573-7.

16. Lobbezoo F, Naeije M. Bruxism is mainly regulated centrally, not peripherally. J Oral Rehabil 2001 Dec;28(12):1085-91.

17. Belser UC, Hannam AG. The influence of altered working-side occlusal guidance on masticatory muscles and related jaw movement. J Prosthet Dent 1985 Mar;53(3):406-13.

18. Sugimoto K, Yoshimi H, Sasaguri K, Sato $\mathrm{S}$. Occlusion factors influencing the magnitude of sleep bruxism activity. Cranio 2011 Apr;29(2):127-37.

19. Sari S, Sonmez H. The relationship between occlusal factors and bruxism in permanent and mixed dentition in Turkish children. J Clin Pediatr Dent 2001 Spring;25(3):191-4.

20. Yustin D, Neff P, Rieger MR, Hurst T. Characterization of 86 bruxing patients with long-term study of their management with occlusal devices and other forms of therapy. J Orofac Pain 1993 Winter; 7(1):54-60.

21. Tsukiyama Y, Baba K, Clark GT. An evidence-based assessment of occlusal adjustment as a treatment for temporomandibular disorders. J Prosthet Dent 2001 Jul;86(1):57-66.
CALDERAN MF SILVA TC HONÓRIO DR oliveira tM MACHADO MAAM

FATORES ETIOLÓGICOS DO BRUXISMO DO SONO: REVISÃO DE Literatura
REV, ODONTOL,
UNIV, CID. SÃO
PAULO
2014; $26(3): 243-$
9, SET-DEZ 
CALDERAN MF

SILVA TC

HONÓRIO DR

OLIVEIRA TM:

MACHADO MAAM

FATORES

ETIOLÓGICOS

DO BRUXISMO DO

SONO: REVISÃO DE

LITERATURA

248
I SSN 1983-5183

22. Manfredini D, Visscher CM, Guarda-Nardini L, Lobbezoo F. Occlusal factors are not related to self-reported bruxism. J Orofac Pain 2012 Summer;26(3):163-7.

23. Steriade M. Arousal: revisiting the reticular activating system. Science 1996 Apr 12;272(5259):225-6.

24. Garcia-Rill E. Disorders of the reticular activating system. Med Hypotheses 1997 Nov;49(5):379-87.

25. Lavigne GJ, Kato T, Kolta A, Sessle BJ. Neurobiological mechanisms involved in sleep bruxism. Crit Rev Oral Biol Med 2003 14(1):30-46.

26. Carra MC, Rompre PH, Kato T, Parrino L, Terzano MG, Lavigne GJ, et al. Sleep bruxism and sleep arousal: an experimental challenge to assess the role of cyclic alternating pattern. J Oral Rehabil 2011 Sep;38(9):635-42.

27. Lavigne GJ, Huynh N, Kato T, Okura K, Adachi K, Yao D, et al. Genesis of sleep bruxism: motor and autonomiccardiac interactions. Arch Oral Biol 2007 Apr;52(4):381-4.

28. Kato T, Montplaisir JY, Guitard F, Sessle BJ, Lund JP, Lavigne GJ. Evidence that experimentally induced sleep bruxism is a consequence of transient arousal. J Dent Res 2003 Apr;82(4):284-8.

29. Gordis EB, Granger DA, Susman EJ, Trickett PK. Salivary alpha amylasecortisol asymmetry in maltreated youth. Horm Behav 2008 Jan;53(1):96103.

30. Gatti R, Antonelli G, Prearo M, Spinella P, Cappellin E, De Palo EF. Cortisol assays and diagnostic laboratory procedures in human biological fluids. Clin Biochem 2009 Aug;42(12):120517.

31. Obayashi K. Salivary mental stress proteins. Clin Chim Acta 2013 Oct 21;425(196-201.
32. Lobbezoo F, Lavigne GJ, Tanguay R, Montplaisir JY. The effect of catecholamine precursor L-dopa on sleep bruxism: a controlled clinical trial. Mov Disord 1997 Jan;12(1):73-8.

33. McCarley RW. Neurobiology of REM and NREM sleep. Sleep Med 2007 Jun;8(4):302-30.

34. Vanderas AP, Menenakou M, Kouimtzis T, Papagiannoulis L. Urinary catecholamine levels and bruxism in children. J Oral Rehabil 1999 Feb;26(2):103-10.

35. Manfredini D, Lobbezoo F. Role of psychosocial factors in the etiology of bruxism. J Orofac Pain 2009 Spring;23(2):153-66.

36. Winocur E, Uziel N, Lisha T, Goldsmith C, Eli I. Self-reported bruxism - associations with perceived stress, motivation for control, dental anxiety and gagging. J Oral Rehabil 2011 Jan;38(1):3-11.

37. Winocur E, Gavish A, Voikovitch $M$, Emodi-Perlman A, Eli I. Drugs and bruxism: a critical review. J Orofac Pain 2003 Spring;17(2):99-111.

38. Sabuncuoglu O, Ekinci O, Berkem M. Fluoxetine-induced sleep bruxism in an adolescent treated with buspirone: a case report. Spec Care Dentist 2009 Sep-Oct;29(5):215-7.

39. Gerber PE, Lynd LD. Selective serotonin-reuptake inhibitor-induced movement disorders. Ann Pharmacother 1998 Jun;32(6):692-8.

40. Monti JM. Disturbances of sleep and wakefulness associated with the use of antihypertensive agents. Life Sci 1987 Oct 26;41(17):1979-88.

41. Tan E-K, Jankovic J, Ondo W. Bruxism in Huntington's disease. Mov Disord 2000 15(1):171-3.

42. Silvestri R, Gagliano A, Arico I, Calarese T, Cedro C, Bruni O, et al. Sleep disorders in children with Attention-Deficit/Hyperactivity Disorder (ADHD) recorded overnight by videopolysomnography. Sleep Med 2009 Dec;10(10):1132-8. 
43. Srivastava T, Ahuja M, Srivastava M, Trivedi A. Bruxism as presenting feature of Parkinson's disease. J Assoc Physicians India 2002 Mar;50(457.

44. Lavigne GL, Lobbezoo F, Rompre PH, Nielsen TA, Montplaisir J. Cigarette smoking as a risk factor or an exacerbating factor for restless legs syndrome and sleep bruxism. Sleep 1997 Apr;20(4):290-3.

45. Lindqvist B. Bruxism in twins. Acta Odontol Scand 1974 32(3):177-87.

46. Carlsson GE, Egermark I, Magnusson
T. Predictors of bruxism, other oral parafunctions, and tooth wear over a 20-year follow-up period. J Orofac Pain 2003 Winter;1 7(1):50-7.

47. Faulkner KD. Bruxism: a review of the literature. Part I. Aust Dent J 1990 Jun;35(3):266-76.

48. Macedo CR, Silva AB, Machado MA, Saconato H, Prado GF. Occlusal splints for treating sleep bruxism (tooth grinding). Cochrane Database Syst Rev 2007 4):CD005514.

Recebido em 29/10/2014

Aceito em 11/03/2015
CALDERAN MF

SILVATC

HONÓRIO DR

OLIVEIRA TM

MACHADO MAAM

FATORES

ETIOLÓGICOS DO

$B R U X I S M O D O$

SONO: REVISÃO DE

LITERATURA 